\title{
Implementation of a hybrid distributed/centralized real-time monitoring system for a DC/AC microgrid with energy storage capabilities
}

\author{
Pablo García, Member, IEEE, Pablo Arboleya, Senior Member, IEEE, Bassam Mohamed, Member, IEEE, Abel \\ Alberto Cuadrado Vega, Member, IEEE and Marcos Cuadrado Vega
}

\begin{abstract}
This paper proposes a combined distributed/centralized architecture for the control and monitoring of a hybrid DC/AC microgrid with energy storage capabilities. The monitoring system is based on an own developed $\mathrm{C}++$ framework for the measurement and real-time state estimation of the microgrid. Calculations running at sampled values (SV) frequency $(10 \mathrm{kHz})$ are implemented into a set of distributed measurement and processing units which have a TCP/IP communication link with a central server running the powerflow (PF) algorithm. The architecture is fully scalable, with the only restrictions of the signal processing capabilities of the distributed units, LAN bandwidth and central server calculation capabilities. The proposed system includes the hardware and software architecture for the monitoring of the microgrid, the communications scheme, the implementation of real-time algorithms for grid state estimation, a graphical user interface including different visualization alternatives and data storing/retrieving capabilities.
\end{abstract}

\section{INTRODUCTION}

$\mathbf{T}$ HE increased use of distributed generation (DG) is changing the approach for distribution networks (DNs) planning and operation. Traditionally, the DNs connected the transmission network with the electricity end users. Power plants were accommodated at transmission level according with a centralized generation paradigm. Under this scenario, the DNs management and operation can be easily guaranteed due to a unidirectional upstream to downstream $\mathrm{PF}$ and communication links. In the last years, a general increasing of the environmental consciousness and a reduction of the DGs costs have driven a change from the centralized to the distributed generation approach. The installation of generators at distribution level could bring several advantages like the reduction of the losses, the increase of the power quality or the possibility of offering ancillary services like voltage or frequency regulation at distribution level [1], [2]. However, if the DGs are installed in an uncoordinated way, the effect could be the opposite [3]. In the recent years, there is a common agreement on the use of microgrids (MGs) with advanced

This work has been partially funded by the Research, Technological Development and Innovation Program Oriented to the Society Challenges of the Spanish Ministry of Economy and Competitiveness under grant ENE2013-44245-R and by the European Union through ERFD Structural Funds (FEDER).

The authors are with the Department of Electrical and Computer Engineering, University of Oviedo, Gijón 33204, Spain (e-mail: garciafpablo@uniovi.es; arboleyapablo@uniovi.es; mohamedbassam@uniovi.es; cuadradoabel@uniovi.es; marcoscv@isa.uniovi.es).

Copyright (c) 2009 IEEE. Personal use of this material is permitted. However, permission to use this material for any other purposes must be obtained from the IEEE by sending a request to pubs-permissions@iee.org communication capabilities, or so called smartgrids (SGs), for the future DN planning [4]-[7].

The MG and SG allow for a topological division of the DN in smaller dispatchable units, with local energy generation and consumption, but also with controllable boundaries for the exchange of energy with neighborhood systems [4]. However, for the massive implementation of this approach, two key technologies must be fully developed: 1) communication systems (CS) [7]-[10], which allow for the collaborative operation of all the grid elements, and 2) energy storage systems (ESS) [11]-[14], for mitigating the impact of the stochastic nature of most of the renewable generation systems.

CS have to be designed considering that a resilient communication link between the producers/consumers/prosumers is needed. The communication links among the different stakeholders can have different specifications, depending on the information to be exchanged, the data rate and the synchronization needs [15]. A remarkable difference with the needs for the centralized grid, is the increased importance of bidirectional communications [10]. It is also relevant the effort made for the standardization [16]. Modbus, originally developed for the communications on process control systems, has been "de facto" standard. However, a more modern approach has been proposed by the International Electrotechnical Commission (IEC) under the IEC 61850 standard. More specifically, the IEC 61850-7-420 (Basic communication structure - Distributed energy resources logical nodes) and the IEC 61850- 90-7 (Advanced Power System Management Functions and Information Exchanges for Inverter-based DER Devices) [17]-[19].

ESS must be incorporated into the grid for increasing the reliability of the energy supply under variant ambient conditions and in order to balance the differences between the produced and the consumed energy. The different ESS strategies can be summarized into three categories: 1) central, 2) distributed and, 3) hybrid [20], [21].

The proposed grid architecture for this work relies on a hybrid orientation. The traditional distribution center is retrofitted by using a three-port solid state transformer (3PSST) [22]. A central ESS is connected to one of the three ports (see Fig. 1). The other two ports are connected to the conventional distribution grid and to a DC link in which a number 
of DC/AC converters allows to keep the same distribution scheme as used nowadays, but with a completely controllable bidirectional PF. At the different points of connection for the consumers/prosumers, the existence of smaller local ESS (dESS) is considered.

This work proposes a distributed monitoring system for a hybrid DC/AC MG, based on the local measurements of currents and voltages at each of the grid nodes and on distributed real-time signal processing on the frequency domain for the isolation of the sequence components, positive/negative/zero, (pnz). The distributed control system has a communication link with the central server at a relatively low sample rate (200ms) and with moderated specifications for the communications bandwidth $(80 \mathrm{~kb} / \mathrm{s})$. At the aforementioned server, a Backward/Forward Sweep (BFS) Power Flow algorithm [23], the grid state visualization and the central data storage are considered. The proposed architecture has been tested in a laboratory environment with a reduced number of nodes.

\section{SYSTEM OVERVIEW}

The proposed MG structure is shown in Fig. 1. As shown, the system is formed by a single interface with the main $\mathrm{AC}$ grid. An integrated 3P-SST, whose internal structure is shown in Fig. 2, with one port connected to the high-side DC link, the second one connected to a central ESS (Li-Ion battery) and the third port to the low-side DC link. Connected to the low-side DC link there are two independent 4-wire inverters, namely head nanogrid converter (HNGC), providing the AC distribution downstream to the so called nanogrid $(n G)$. Each of the $n G$ has two additional nodes, representing buildings, to which loads, renewable/dispatchable $(R G / D G)$ generation and dESS can be connected. Main electrical features for the MG are shown in Table I.

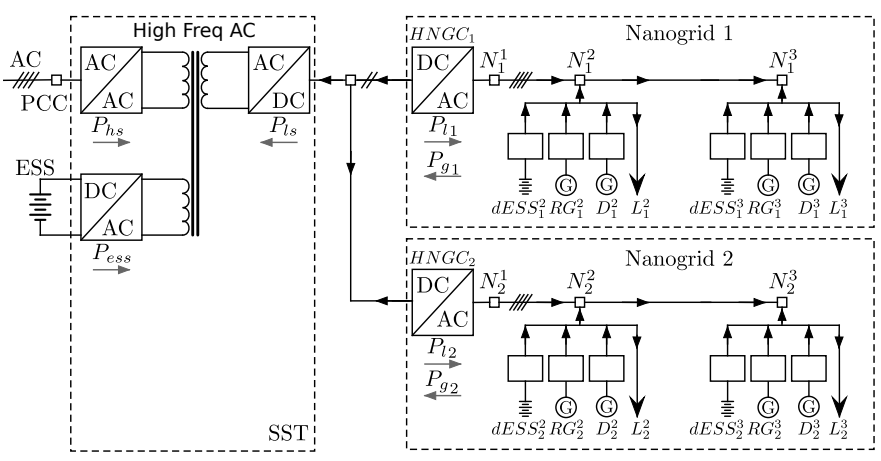

Fig. 1. Proposed MG structure. The 3P-SST allows for the exchange of power among the different grid points (AC mains, centralized ESS and AC $n G$ ). Each of the $n G$ is powered by a 4-wire HNGC. The internal nodes $\left(N_{x}^{y}\right)$ of the two $n G$ represent buildings to which the different loads $\left(L_{x}^{y}\right)$, DG (both renewable, $R G_{x}^{y}$, and dispatchable, $D_{x}^{y}$ ) and dESS are connected.

The main goals of the proposed system architecture are to achieve near zero energy from the main AC grid, to increase the reliability and to isolate the effect of grid disturbances and faults from one $n G$ to the other. Each individual building is also configured to minimize the energy demand from the AC $n G$, by using its own local generation and energy storage. In order to minimize the effects of unbalanced loads or generation, each building will also try to reduce the impact of its own

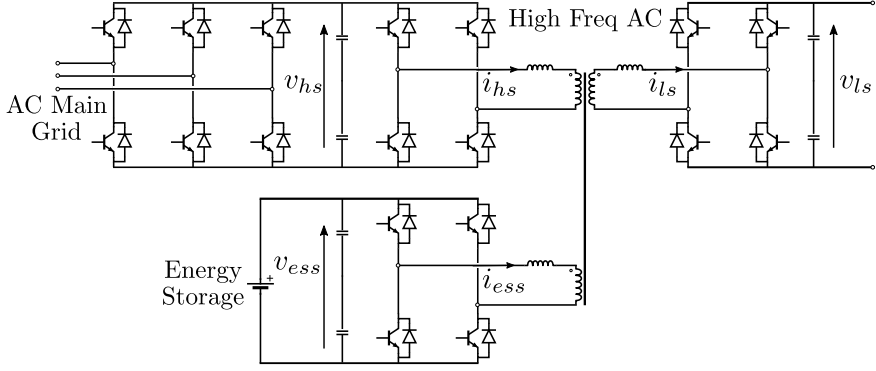

Fig. 2. 3P-SST internal structure and variables used for the control implementation.

TABLE I

GRID PARAMETERS

\begin{tabular}{c|c}
\hline Parameter & Nominal value \\
\hline & Header Converter (PCC) \\
\hline Power & $150 \mathrm{kVA}$ \\
Legs/Wires & $6 / 3$ (possible parallel operation) \\
Switching Freq & $10 \mathrm{kHz}$ \\
\hline \multicolumn{2}{c}{ Three-Port Solid State Transformer } \\
\hline Power & $150 \mathrm{kVA}$ \\
Switching Freq & $20 \mathrm{kHz}$ \\
Port 1 & Header Converter 900 Vdc \\
Port 2 & ESS. Li-Ion Battery, 16kWh/48kW. 375 Vdc \\
Port 3 & Inner Microgrid. Low DC link 750 Vdc \\
\hline \multicolumn{2}{c}{ Nanogrid Converters } \\
\hline Power & $50 \mathrm{kVA}$ \\
Switching Freq & $10 \mathrm{kHz}$ \\
Legs/Wires & $4 / 4$ \\
\hline
\end{tabular}

created negative/zero-sequence currents and, when exceeding energy is available, to use that remaining capability to provide help in the compensation of external created unbalances. In order to achieve this, the modes of operation of the 3P-SST and the AC $n G$ are described in III-A and III-B respectively.

In order to implement the control and monitoring of the MG, the hardware architecture shown in Fig. 3 has been implemented. For the 3P-SST, the three DC-link voltages and port output currents are measured. At each node at the AC side (HNGC, buildings) three voltages (phase to neutral) and three/four currents (line and optionally the neutral) are measured. The measured signals are interfaced to a digital signal controller (DSC, Texas Instruments F28M35) which is connected through an Ethernet interface to a Raspberry Pi $\mathrm{B}+$ model. The data processed at the Raspberry Pi is sent to the central control system using another Ethernet interface. The central control system runs the state estimation algorithm, shows the graphical user interface and stores the processed variables in a database.

\section{SYSTEM CONTROL}

\section{A. 3P-SST Control}

The 3P-SST is responsible for controlling the voltage at the low-side DC-link to which the two DC/AC converters distributing $\mathrm{AC}$ energy to the $n G$ are connected. The energy can flow following different paths, which correspond to the different operating modes.

The proposed system control scheme is summarized at Fig. 4. The low-side DC-link voltage, $v_{l s}$, is controlled using a cas- 


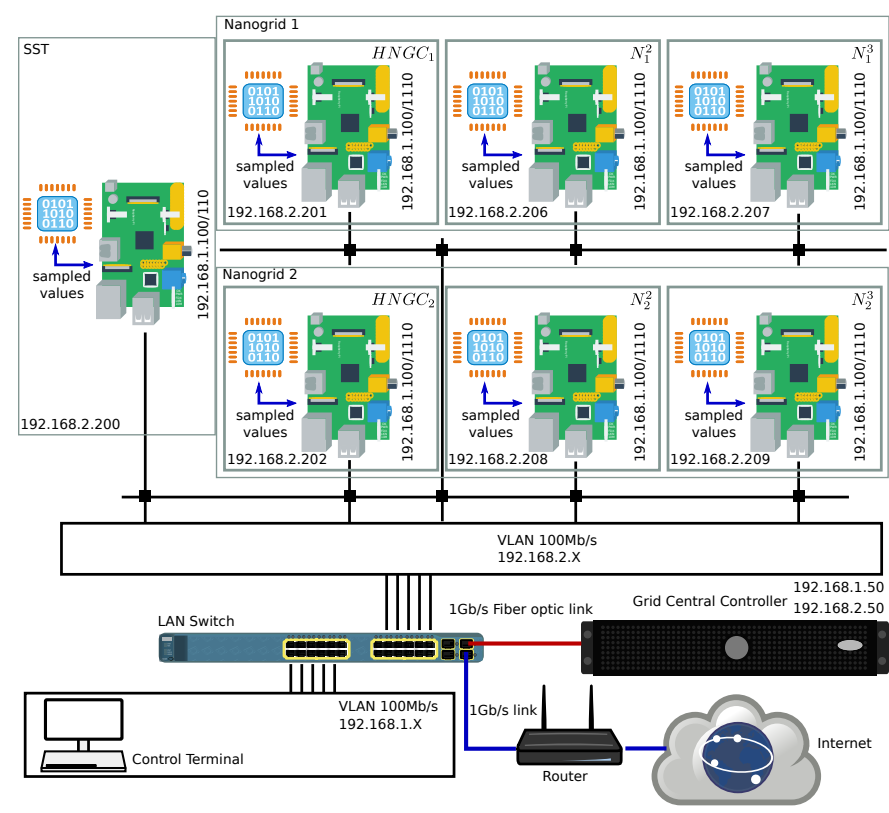

Fig. 3. Communications network for the test set up. Each of the grid nodes has a Raspberry Pi interfaced with the different shunt control systems by a private TCP/IP connection (192.168.1.100 to 192.168.1.110 internal IP range). The interface with the central server is done by a $100 \mathrm{Mb} / \mathrm{s}$ VLAN (192.168.2.X). The central controller is interfaced with the LAN switch by a $1 \mathrm{~Gb} / \mathrm{s}$ direct fiber optic link. The system can be accessed for control purposes both from an internal separated VLAN (192.168.1.X) or from an external internet connection.

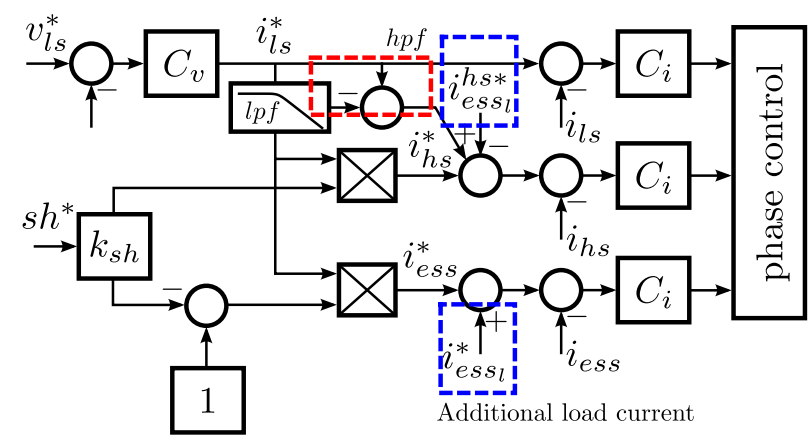

Fig. 4. Proposed control for the 3P-SST. The three ports are current controlled $\left(C_{i}\right)$ for improved dynamics. The low-side DC link voltage is the external loop $\left(C_{v}\right)$. The sharing for the power (current) demand from the AC mains $\left(i_{h s}^{*}\right)$ or the ESS $\left(i_{\text {ess }}^{*}\right)$ can be adjusted by the $s h^{*}$ reference and the $k_{s h}$ constant.

caded control loop strategy, imposing an inner current/power control loop, $i_{l s}$. The reference for the circulating current at the low-side transformer winding, $i_{l s}^{*}$, is then dynamically shared between the high-side, $i_{h s}^{*}$, and the ESS, $i_{\text {ess }}^{*}$, ports, which are also current/power-controlled. In order to meet the power constraint at the ESS, the sharing reference is lowpass filtered, being the complementary current added to the high-side reference, which will be then supplying the system during high-frequency transients. The selection of the cut-off frequency and frequency order depends on the ESS power rating specifications, and are not considered in this paper. The sharing mechanism is implemented by the $k_{s h}$ gain, which can be online updated based on the $s h^{*}$ command. The command is given by the high-level control loops [22] and could be changed considering different operation constraints such as:
1) economical aspects, 2) reliability considerations and 3) maintenance operation. The $k_{s h}$ gain is a number between $[-1,1]$, leading to the different operation modes:

1) mode\#1. $k_{s h}=1$, makes the ESS not to participate in the power exchange. All the power is transferred between the $\mathrm{AC}$ mains at the $\mathrm{AC} n G$. In the case there is exceeding local generation at the $n G$, the surplus power is returned back to the AC mains.

2) mode\#2. $k_{s h}=0$, makes the SST and the AC $n G$ to be disconnected from the AC mains. All the power exchange occurs between the ESS and the $\mathrm{AC} n G$. When the $\mathrm{AC} n G$ local generation is higher than the corresponding loads, the current is returned back to the ESS, thus allowing for the charging operation.

3) mode\#3. $k_{s h}=[0,1]$, both the AC mains and the ESS participate in the power exchange, with a weight proportional to the value of $k_{s h}$. Direction of PF at the high-side is equal to the $\mathrm{PF}$ direction at the low-side.

4) mode\#4. $k_{s h}=[-1,0]$, both the $\mathrm{AC}$ mains and the ESS participate in the power exchange, with a weight proportional to the value of $k_{s h}$. Direction of PF at the high-side is opposed to the PF direction at the low-side.

In addition to the enumerated modes, the ESS can be at any moment charged/discharged from/to the AC grid, being that represented by the additional reference given at the ESS port, $i_{\text {essl }}^{*}$, and the corresponding current at the high-side port, $i_{\text {ess } l}^{h s *}$. This enables to assert a safe state of charge of the device under any condition (providing the connection to the $\mathrm{AC}$ mains is available).

The performance of an averaged model for the different load conditions and $k_{s h}$ values is shown at Fig. 5 and here explained: 1) initially $(t=0 s)$ the low-side DC link is discharged, being the needed active power demanded from the AC grid, 2) at $t=2 s$, a load is connected at the low-side DC link, thus representing a power demand at the $\mathrm{AC} n G . k_{s h}$ is set to 1 and so all the needed power is demanded from the AC grid. 3) at $t=4 s, k_{s h}$ is set to 0 , thus moving the power demand from the AC main grid to the ESS. From this instant, the internal distribution system could be decoupled from the mains, working in autonomous mode. This could be applied based on time-shifting strategies in order to take advantage of reduced tariff or because of any contingency at the main $\mathrm{AC}$ grid. 4) at $t=6 s$, local generation at the $\mathrm{AC} n G$ starts, the exceeding power is automatically handled by the low-side DC link voltage controller and moved to the ESS, thus starting the recharge of the battery. 5) at $t=8 \mathrm{~s}$, ESS increases its charge rate by demanding more active power, the power is absorbed from the AC grid. During the transients, particularly noticeable at $t=6 \mathrm{~s}$, it can be seen the complementary power supply by the high-side and ESS ports. By changing the cut-off frequency of the filter shown at Fig. 4, the transient sharing can be easily varied, thus increasing the flexibility of the proposed system.

\section{B. AC nanogrid Control}

Each HNGC connected to the DC bus feeds one $n G$ and has installed a $n G$ central controller. The references to that controller are provided from the grid central controller, as 

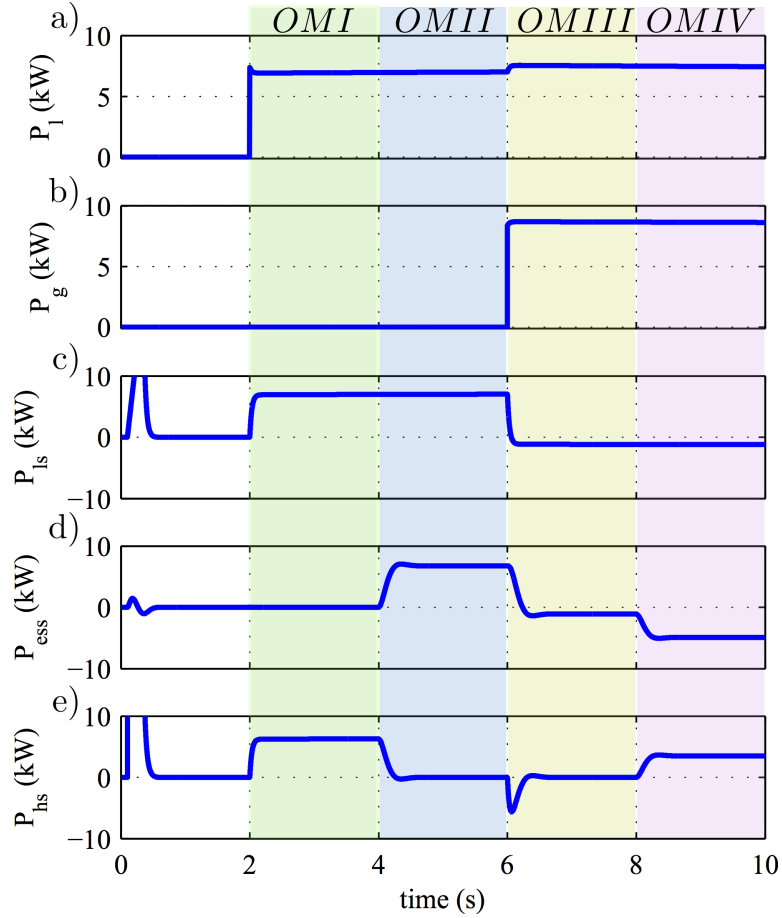

Fig. 5. Resulting powers for different operation modes (OM at the figure) From top to bottom, a) load power at low-side, b) generation power at lowside, c) total low-side power, d) ESS power, e) high-side power. The initial transient shown at $t=0$ is for the charging of the DC link and it is not considered during the regular operation of the system.

depicted in Fig. 3. In each $n G$, the nodes represent different buildings. Each building can have installed dispatchable and renewable generation as well as dESS and loads. Such devices can be connected to the $\mathrm{AC} n G$ both using three/four wires converters.

At building level, the Building Management Systems (BMS) collects the measurements from the converters and the loads installed in the building and sends the information to the grid central controller. This report must include, at least, the information about the voltages (RMS) at the node and the net powers absorbed or injected from/to the network by the building. Additionally, in the case it is available, it could contain phasor information about all voltages and currents. From the point of view of the control system, there is a substantial difference for each approach. In the case phasors are needed, synchronization for the measurements among all the nodes is required. When RMS values are used instead, there is no need for synchronization. The static constraints at building level, like available renewable power, stored energy, availability of the generators, etc, are stored at the central controller database. In the event of any change of these parameters, the BMS will also notify the central controller.

For the estimation of each $n G$ state, the central grid controller firstly runs a PF algorithm. Then, an optimal power flow (OPF) algorithm is used to calculate the optimal set points for building converters, in order to reduce the total losses in the system and the dependance from the main grid. In this OPF, the data and constraints provided by all the BMSs on a $n G$ are put together with the data from the other $n G$ as well as from the SST interface. Finally, the grid central controller sends the calculated set points to the HNGC and all the BMSs.

\section{MICROGRID MONITORING SYSTEM}

\section{A. System architecture}

The software architecture is split into three main components: 1) signal acquisition, 2) distributed signal processing, $3)$ central signal processing. The signal acquisition is carried out at the DSC used for low-level control purposes of the MG converters (Texas Instruments F28M35). Besides the control tasks, the system is able to send the sensors' data to the Raspberry Pi using an embedded TCP/IP Stack (uIP [24]). The distributed signal processing, running at the Raspberry $\mathrm{Pi}$ installed at each node, and the central signal processing, running at the MG central controller, relies on the same own developed C++ framework, namely FPS. The specific MG architecture ( $n G /$ lines/buses/shunt objects) is loaded from a MySQL database, and the different objects and signals are dynamically generated from this database configuration data. The key elements stored at the database, with their respective inheritance diagram, are shown in Fig. 6. The functionality for each object is as follows:

1) Group Info. Base class for the database representation of signals which belong to a same group.

a) Line Info. Class for the representation of a grid line.

b) Shunt Info. Base class for the representation of an electrical element connected in shunt.

i) Shunt Generator Info. Class specification for generators connected to the system.

A) Dispatchable Info. Class for the codification of a dispatchable generator.

B) Renewable Info. Class for the codification of a renewable generation system.

C) ESS Info. Class for the codification of an ESS.

ii) Shunt Load Info. Class specification for loads connected to the system.

c) Bus Info. Class for representing a grid node.

d) NanoGrid Info. Class for representing an AC nanogrid.

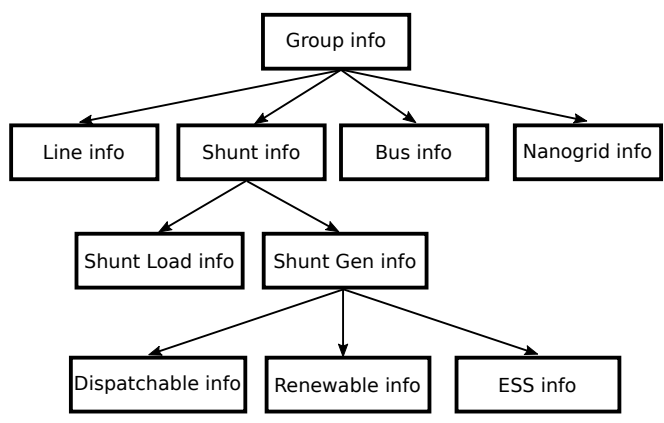

Fig. 6. Key elements stored at the database. The inheritance for each object is represented at the database by specific table relations. When loading or saving the information from/to the database, the FPS framework design makes each object to deal with its own data fields following the same inheritance diagram.

The system elements presented in Fig. 1 are translated to an object oriented design as follows. Any node in the system is represented by an instance of the Bus Grouper class and 


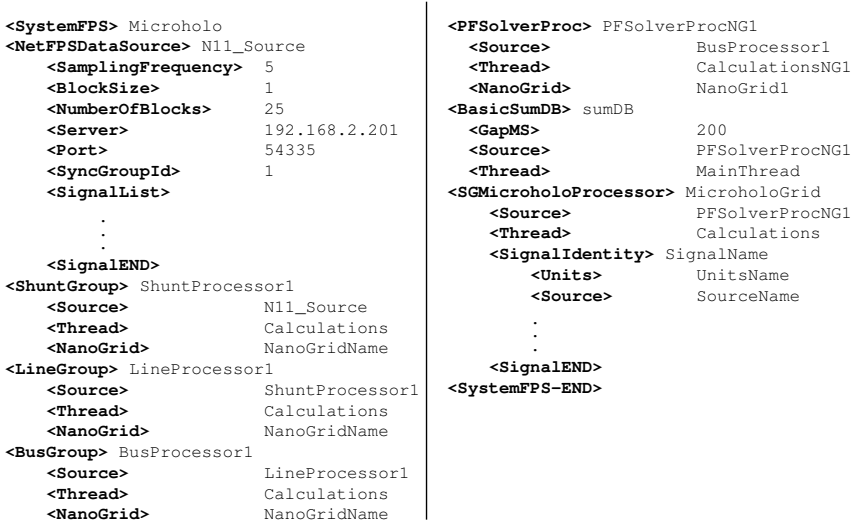

Fig. 7. Example for a portion of the configuration file used at the developed framework.

the distribution lines between two nodes are translated to a Line Grouper class. Any system connected to the node inherits from a base Shunt Grouper class and has its own specialized representation, namely Load, ESS, RG and DG for loads, energy storage systems, renewable generation and distributed generation respectively. System topology is created from a file, also stored at the database, containing the objects connections and the configuration. An example for a simple configuration file is given at Fig. 7. Calculations are done at each object using a synchronous approach, as shown in Fig. 8. The sample time is given by the sample time of the sources, and for this application is $10 \mathrm{kHz}$. The individual samples are packed in blocks of data and, as soon as the block is full and an Ask Data message has been received, a Data Ready message is sent to all the connected objects downstream. For this application, a block size of 2000 samples has been selected, leading to a $200 \mathrm{~ms}$ update rate. When all the connected sources to a given object have notified the Data Ready message, the calculation method Update is invoked and the resulting data is obtained. This procedure is repeated until the end of the chain is reached. When the final elements are done with the calculations for the actual block, a new message Ask Data is sent from the sinks to the sources and the procedure starts again for the next block. In the case the time needed for the operations of all the objects in a chain is greater than the block time (200 $\mathrm{ms}$ for this application), the source block is discarded. All the blocks are marked with a time stamp having $1 \mathrm{~ms}$ resolution which allows to determine when the data has been generated or calculated.

The data produced or calculated at each data block is internally assigned to a Signal, which are the data carriers. Each element at the signal processing chain will offer a variable number of signals to the connected objects. Each signal will internally have a reference to its origin signal/s, thus allowing to easily iterate over all the signals in an object for reading the origin data and performing the needed calculations.

Each of the individual objects shown can be executed in its own thread, allowing for a better distribution of the processor resources. Synchronization among the different threads is achieved by using mutex resources. The application main thread is the responsible for the graphical user interface.

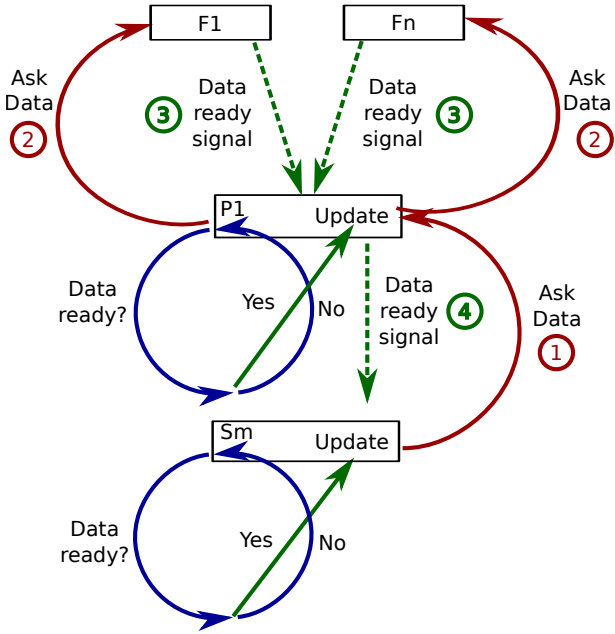

Fig. 8. System events and states. $F_{x}$ labels are used for data sources, $P_{y}$ for data processors and $S_{z}$ for data sinks. Reading of data at each object is done at the Update method. Ask Data signals are emitted from the sinks to the sources, whereas Data ready signals are from the sources to the sinks. Numbers indicate the sequence order in the messaging system.

\section{B. Processing units}

In order to calculate the PF in the microgrid and the state estimation algorithm, the time domain signals are translated to the $p n z$ domain from where all the calculations are done. A flow diagram for the signal processing is shown in Fig. 9. The different steps are here summarized:

- Net Source FPS. The three phase voltages $\left(v_{a n}^{i}, v_{b n}^{i}, v_{c n}^{i}\right)$ and four currents $\left(i_{a}^{i}, i_{b}^{i}, i_{c}^{i}, i_{n}^{i}\right)$ for each node $(i)$ are acquired using the DSC analog to digital interface. The raw signals are sent to the corresponding Raspberry Pi for the distributed signal processing. Details on the communications protocol are given in VI.

- Clarke. The $\alpha \beta 0$ components for the voltages $\left(v_{\alpha}^{i}, v_{\beta}^{i}, v_{0}^{i}\right)$ and the currents $\left(i_{\alpha}^{i}, i_{\beta}^{i}, i_{0}^{i}\right)$ are obtained by using the transformation matrix (2).

- ReImg2Complex. The complex voltage $\left(\mathbf{V}_{\alpha, \beta}^{i}\right)$ and current $\left(\mathbf{I}_{\alpha, \beta}^{i}\right)$ vectors at the stationary reference frame are built from their components.

- Sequence Extractor. A sequence analyzer block is used to obtain the positive $\left(\mathbf{V}_{p}^{i}, \mathbf{I}_{p}^{i}\right)$, negative $\left(\mathbf{V}_{n}^{i}, \mathbf{I}_{n}^{i}\right)$ and zero $\left(\mathbf{V}_{z}^{i}, \mathbf{I}_{z}^{i}\right)$ sequences. A frequency-based algorithm is used in order to also allow for getting the signals harmonics. The method is based on the Goertzel algorithm [25] and it uses 10 fundamental cycles $\left(200 \mathrm{~ms}\right.$ at $f_{e}=50 \mathrm{~Hz}$ ) for getting a spectral resolution of $5 \mathrm{~Hz}$ on the calculated harmonics. The number of samples has been chosen as a tradeoff between the spectral resolution and the calculation speed. The positive, negative and zero sequences for the voltages and the currents are used to calculate the different power expressions:

- PQ_al_be_harm. Positive/Negative/Zero sequence active/reactive powers.

- PQabc. Phase Active/Reactive Powers.

- PNZ2abc. Voltage/current phasors.

- Shunt Grouper. The signals are grouped by shunt elements 


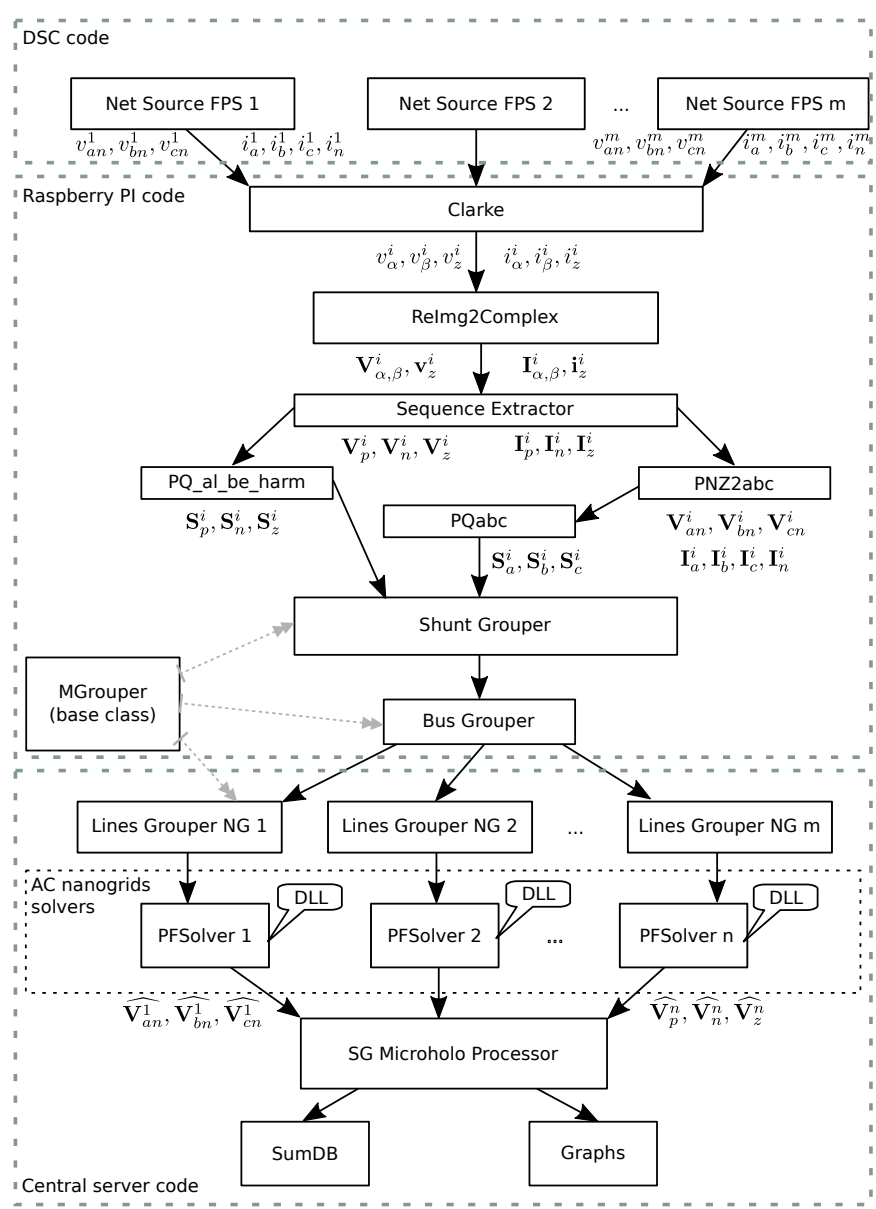

Fig. 9. Signal processing chain.

using a tag in one of their properties. This will allow to determine the data source once the information arrives to the central server.

- Communication link with the central server. The calculated voltages, currents and powers at each shunt device are transmitted to the central server using a TCP/IP link. Details on the communications protocol are given in VI.

- Bus Grouper. The different shunt signals transmitted from the distributed units are grouped together at bus level. The groups are created by adding a bus tag to the tag property of each signal. Additionally, the overall current and voltage at each bus are calculated using the sign convention pointed by the arrows in Fig. 1.

- Lines Grouper NG $\mathbf{x}$. The signals corresponding to each line are grouped together using a tag property at each signal. The resulting line currents are calculated following the sign convention shown in Fig. 1.

- PF Solver $\mathbf{x}$. The PF problem is executed for each individual $\mathrm{AC} n G$. For the present paper, the $\mathrm{AC}$ systems are considered to be independent by assuming a strong DC link voltage control on the $n G$ header converters.

- Microgrid State Processor. The outputs of the PF algorithm and the resulting processed signals acquired from the sensors are here grouped and compared in order to build an estimation of the MG. The final values are represented at
TABLE II

CALCULATIONS TIME

\begin{tabular}{lcc||lcc}
\multicolumn{2}{c}{ RASPBERRY-PI SIDE } & \multicolumn{3}{c}{ SERVER SIDE } \\
\hline & $\begin{array}{c}\text { Mean } \\
(\mathrm{ms})\end{array}$ & $\begin{array}{c}\text { Std } \\
(\mathrm{ms})\end{array}$ & & $\begin{array}{c}\text { Mean } \\
(\mathrm{ms})\end{array}$ & $\begin{array}{c}\text { Std } \\
(\mathrm{ms})\end{array}$ \\
Clarke & 39.9 & 3.9 & DBSink & 6.8 & 2.1 \\
Re-Im2Cmplx & 4.0 & 0.8 & Graphs & 3.2 & 1.9 \\
Seq. Extr. & 6.7 & 1.0 & Line Proc. & 1.4 & 0.9 \\
PQ_al_be_h & 1.4 & 0.8 & MG State Proc. & 10.0 & 3.7 \\
PQabc & 0.7 & 0.5 & PFSolver NG1 & 5.5 & 1.4 \\
pnz2abc & 1.4 & 0.6 & PFSolver NG2 & 2.8 & 0.8 \\
$\quad$ Shunt Proc. & 2.2 & 0.7 & VisualGroup & 2.5 & 3.4 \\
Bus Proc. & 5.0 & 0.9 & & & \\
FPSNet & 5.2 & 0.8 & & &
\end{tabular}

the graphical user interface and sent to the database.

\section{DigITAL SIGNAL PROCESSING IMPLEMENTATION}

The goal of the monitoring system is to effectively obtain a state estimation of the MG using the proposed signal processing chain. All the calculations are done in the time spam of $200 \mathrm{~ms}$. The time required for each individual calculation is later summarized at Table II for the decentralized and centralized calculations respectively. The statistics are calculated for a total of 1000 cycles $(200 s)$. As it can be seen, the total time for the Raspberry Pi signal processing is around $75 \mathrm{~ms}$, whereas for the central controller is below $50 \mathrm{~ms}$. The most important signal processing blocks are following detailed.

\section{A. abc to $\alpha, \beta, 0$}

Using Clarke power invariant transformation [26] (1) $\alpha \beta 0$ values in time domain for the phase voltages and currents are obtained. In the case the neutral current is measured, zerosequence current is directly obtained from the sensor reading. Once the $\alpha \beta 0$ values are obtained, the complex vector required for the sequence extractor is built using $x_{\alpha, \beta}^{i}=x_{\alpha}^{i}+\mathbf{j} x_{\beta}^{i}$.

$$
\mathbf{x}_{[\alpha, \beta, 0]}=\mathbf{A}^{T} \times \mathbf{x}_{[a, b, c]} \quad \mathbf{A}=\sqrt{\frac{2}{3}}\left(\begin{array}{ccc}
1 & 0 & \frac{1}{\sqrt{2}} \\
-\frac{1}{2} & \frac{\sqrt{3}}{2} & \frac{1}{\sqrt{2}} \\
-\frac{1}{2} & -\frac{\sqrt{3}}{2} & \frac{1}{\sqrt{2}}
\end{array}\right)
$$

\section{B. Sequence extractor}

The key block for the signal processing is the sequence extractor, which allows for representing the data in the positive, negative and zero sequences which are later used for the PF Solver. Being the analyzed $n G$ a distribution grid, the system is inherently unbalanced (unbalanced loads and single-phase generation systems). By applying symmetrical components theory [27], the positive, negative and zero sequences can be isolated and the variables can be represented in a sequence space (2),

$$
\mathbf{S}=\frac{1}{\sqrt{3}}\left(\begin{array}{ccc}
1 & 1 & 1 \\
1 & a^{-1} & a^{-2} \\
1 & a^{-2} & a^{-1}
\end{array}\right) \quad \mathbf{x}_{[z, p, n]}^{T}=\mathbf{S} \times \mathbf{x}_{[a, b, c]}^{T}
$$

where $a=e^{j 2 \pi / 3}$. 
The approach given by (2) requires the input signals to be the RMS values for a given fundamental period. However, calculating the RMS value under highly harmonic content, requires the use of low-pass filter elements in order to isolate the fundamental harmonics.

In this paper, an alternative frequency-domain solution is proposed. The system relies on the Goertzel algorithm [25]. The implementation has a lower computational burden when compared with traditional FFT-based approach for a low number of harmonics. Specifically, for calculating $M$ harmonics from an input data vector of length $N$, the associated cost of the Goertzel algorithm can be expressed as $O(N, M)$, whereas for the FFT is $O\left(N, \log _{2} N\right)$. Obviously, when the number of calculated harmonics meets $M \leq \log _{2} N$, then the Goertzel approximation is the preferred choice. In this paper, the number of samples used for the analysis corresponds to $200 \mathrm{~ms}$ of data sampled at $10 \mathrm{kHz}$, thus being $N=2000$. For the proposed application, the calculations using the Goertzel approach are faster when the calculated number of harmonics is $M \leq 10$. For the proposed application, the positive and negative harmonics are calculated from the $x^{i}{ }_{\text {alpha }, \beta}$ complex vector and the positive from the zero-sequence variable. The algorithm implementation is shown in Algorithm 1. At the implementation, the harmonic input variable contains the harmonic order of the sequences being analyzed.

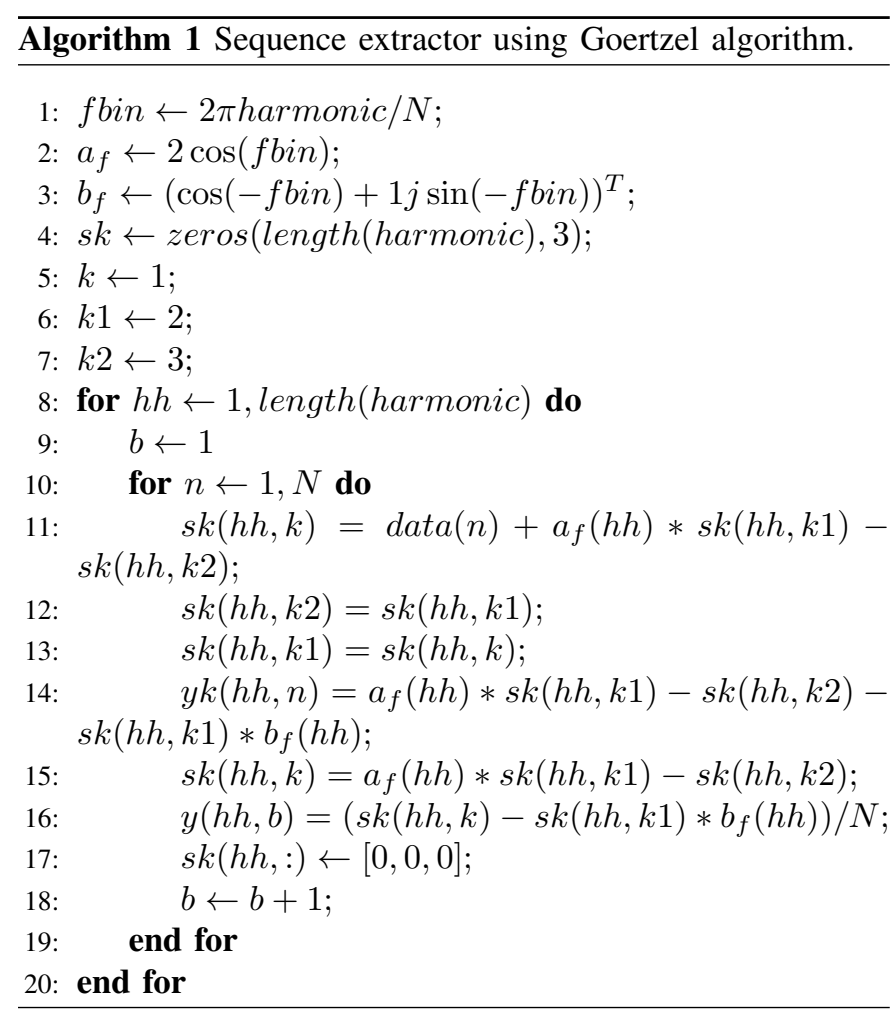

\section{PF Solver}

The PF algorithm and the solver are based on the one developed in [28] and [29] respectively. In those cases the formulation was adapted for using the $\alpha \beta 0$ reference frame, while in the present research the $a b c$ reference has been used instead. The solving procedure is based on the node incidence matrix $\Gamma$. In this matrix, each row represents a line and each column a node. The element $\Gamma_{i j}$ is 1 when the tail of the branch $i$ is node $j$, and -1 when the head of the branch $i$ is node $j . \Gamma_{i j}$ will be 0 otherwise.

Using this matrix formulation, the relations between the microgrid voltages and currents can be expresed in a compact form using (3) to (7).

$$
\begin{array}{lll}
\mathbf{V}_{a b c n}^{B} & =\boldsymbol{\Gamma} \times \mathbf{V}_{a b c n}^{N} \\
\mathbf{V}_{a b c n}^{B} & =\mathbf{Z} \times \mathbf{I}_{a b c n}^{B} \\
\mathbf{I}_{a b c n}^{N} & = & -\boldsymbol{\Gamma}^{\boldsymbol{T}} \times \mathbf{I}_{a b c n}^{B} \\
\mathbf{I}_{a b c n}^{B} & = & -\left(\boldsymbol{\Gamma}^{\boldsymbol{T}}\right)^{-\mathbf{1}} \times \mathbf{I}_{a b c n}^{N} \\
\mathbf{V}_{a b c n}^{N} & =\boldsymbol{\Gamma}^{-\mathbf{1}} \times \mathbf{V}_{a b c n}^{B}
\end{array}
$$

Where $\mathbf{V}_{a b c n}^{N}$ and $\mathbf{V}_{a b c n}^{B}$ represent respectively the phase-toground nodal voltages and voltage drop in the $n G$ branches. In the same way, $\mathbf{I}_{a b c n}^{N}$ and $\mathbf{I}_{a b c n}^{B}$ represent the net current injected in the $n G$ nodes and the currents through the lines (branches). The matrix $\mathbf{Z}$ is the network impedance matrix. It is important to remark that the nodal current in a given node represents the net current demanded in the node, and thus it must be calculated as (8)

$$
\mathbf{I}_{a b c n}^{N}=\mathbf{I}_{a b c n}^{L}-\mathbf{I}_{a b c n}^{A}
$$

being $\mathbf{I}_{a b c n}^{L}$ the current consumed by the loads of the building and $\mathbf{I}_{a b c n}^{A}$ the aggregated current injected by the DGs installed inside the building. This current will be provided in real time by the converters measurement equipment. For the case of the current load, it will be considered as a constant PQ load model according to (9) to (12).

$$
\begin{array}{lll}
\mathbf{S}_{a b c}^{L} & = & \mathbf{P}_{a b c}^{L}+\boldsymbol{j} \cdot \mathbf{Q}_{a b c}^{L} \\
\mathbf{V}_{a b c}^{L} & = & \mathbf{V}_{a b c n}^{N}-\mathbf{V}_{n}^{N} \\
\mathbf{I}_{a b c}^{L} & = & \operatorname{Conj}\left(\mathbf{S}_{a b c}^{L} / \mathbf{V}_{a b c}^{L}\right) \\
\mathbf{I}_{n}^{L} & = & -\sum \mathbf{I}_{a b c}^{L}
\end{array}
$$

Where $\mathbf{S}_{a b c}^{L}, \mathbf{P}_{a b c}^{L}, \mathbf{Q}_{a b c}^{L}$ stand for the load apparent, active and reactive power respectively. $\mathbf{V}_{a b c}^{L}$ and $\mathbf{V}_{n}^{N}$ represent the load phase-to-neutral voltages and the nodal neutral voltage. $\mathbf{I}_{a b c}^{L}$ and $\mathbf{I}_{n}^{L}$ are the load currents through the phases and through the neutral respectively. The variables are graphically represented in Fig. 10. For solving the proposed formulation, a

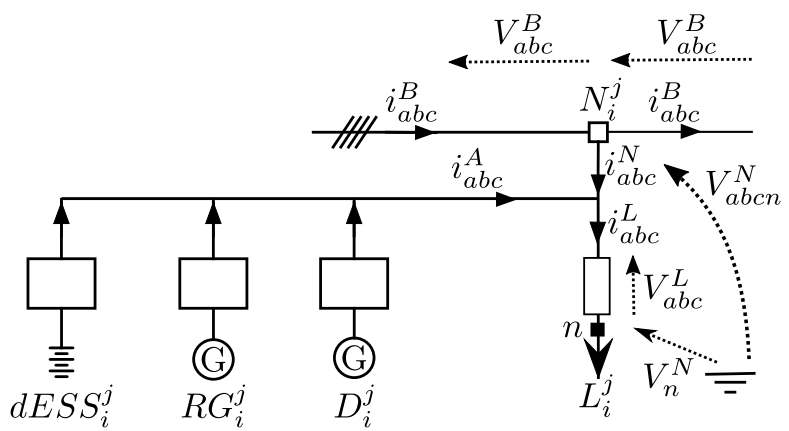

Fig. 10. Schematic representation of the signals used for solving the PF.

Backward/Forward swept algorithm, summarized in Algorithm 
2 , has been used. The voltage provided by the $n G$ head converter will be considered as a slack voltage and will be added to the system of equations as known data.

Algorithm 2 Backward/Forward swept power flow algorithm.
Input: $\mathbf{R}, \mathbf{I}_{0}$
Output: $\mathbf{V}_{a b c n}^{N}$
1: Assume : $\mathbf{V}_{a b c n}^{N}=1$
2: Get load voltage $\mathbf{V}_{a b c}^{L}$ from (10)
3: Get load phase current $\mathbf{I}_{a b c}^{L}$ from (11)
4: Get load neutral current $\mathbf{I}_{n}^{L}$ from (12)
5: Get aggregated current $\mathbf{I}_{a b c n}^{A}$ from converters sensors
6: Get node injection current $\mathbf{I}_{a b c n}^{N}$ from (8)
7: Get branch current $\mathbf{I}_{a b c n}^{B}$ from (6)
8: Get branch voltage $\mathbf{V}_{a b c n}^{B}$ from (4)
9: Get node voltage $\mathbf{V}_{a b c n}^{N}$ from (7)
10: Get $E r r=$ new $\mathbf{V}_{a b c n}^{N}$ - old $\mathbf{V}_{a b c n}^{N}$
11: If $|E r r|>T o l$ then goto step (2)
12: End

\section{COMMUNICATION PROTOCOL}

As stated before, the proposed architecture uses a hybrid approach based on both distributed and centralized calculations. The different parts of the control system are integrated using the communication scheme before described in Fig. 3. The inner control units from the point of view of the system operator are the shunt elements, which are connected to the different grid nodes. In order to have a full set of measurements for the grid state, the minimum amount of variables to sense are the 3 phase voltages and the 4 bus currents. In the case an individual shunt element has additional phase current readings, the variables will be also incorporated to the final variable set. At bus level, a Raspberry Pi interfaced to the different control units is considered. The communication among the control units and the Raspberry Pi is a TCP/IP link using a custom application protocol shown in Fig. 11a). The channels data fields are filled with the A/D sampled values for the grid voltages and phase currents. The A/D resolution is $12 b i t s$ and the sample rate $10 \mathrm{kHz}$.

The communication between each bus and the central MG controller is established by a TCP/IP link between each Raspberry Pi and the central controller. The connections are set in a VLAN in which the only permitted network traffic is that generated by the data exchange protocol. The structure of the datagrams within this communications layer is shown in Fig. 11b). As it can be seen, the protocol allows for a maximum of 128 signals to be sent from each bus, and the individual data fields are considered complex in order to allow the transmission of phasor-related values. The header includes information about the sample rate and the Unix time at the source in order to synchronize the data at the central server. With the calculations time configured for this paper (200ms),
TABLE III

PF RESULTS FOR RMS VOLTAGES AT AC $n G$ NODES

\begin{tabular}{c|ccc||c|ccc}
\multicolumn{7}{c||}{$n G 1$} & \multicolumn{4}{c}{$n G 2$} \\
\hline & $V_{a}-\widehat{V}_{a}$ & $V_{b}-\widehat{V}_{b}$ & $V_{c}-\widehat{V}_{c}$ & & $V_{a}-\widehat{V}_{a}$ & $V_{b}-\widehat{V}_{b}$ & $V_{c}-\widehat{V}_{c}$ \\
\hline$N_{1}^{1}$ & 0 & 0 & 0 & $N_{2}^{1}$ & 0 & 0 & 0 \\
\hline$N_{1}^{2}$ & 0.0550 & 0.0560 & 0.0652 & $N_{2}^{2}$ & -0.1156 & -0.1779 & 0.4480 \\
\hline$N_{1}^{3}$ & 0.0521 & 0.0552 & 0.0690 & $N_{2}^{3}$ & 0.0267 & -0.3086 & 0.4571 \\
\hline
\end{tabular}

and assuming the maximum number of signals per node, the requirement for the network bandwidth is less than $81 \mathrm{~kb} / \mathrm{s}$. The overhead for the header, even with the minimum amount of data sent ( 4 currents and 3 voltages), is kept at $20 \%$.

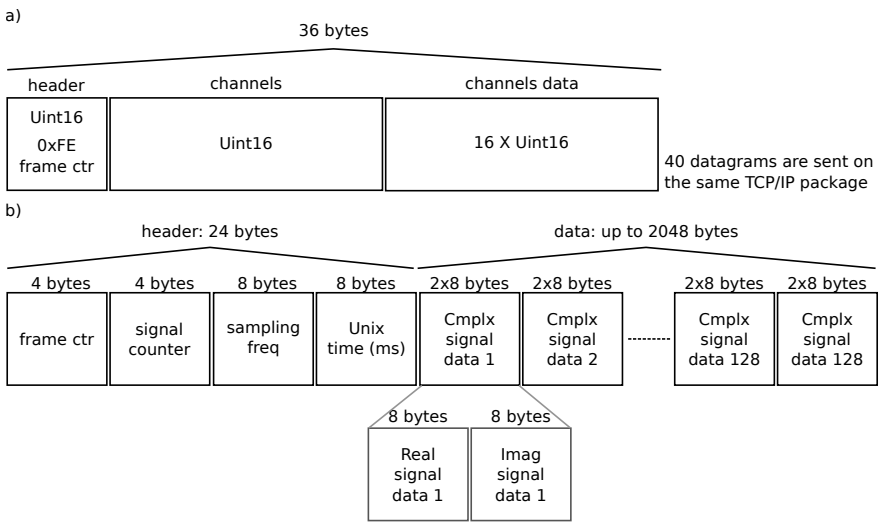

Fig. 11. a) Sampled values data frames for the communication link between the DSC and the Raspberry Pi. The 40 datagrams are filled at the DSC side before building the TCP/IP data packet. Each data packet has a data length of 1440 bytes. b) Processed values protocol for the communication between the Raspberry Pi and the central server. The datagram allows sending complexvalued data for up to 128 signals.

\section{EXPERIMENTAL SETUP}

The test environment, with the running application, is shown in Fig. 12. The input data for the application has been obtained from the smart meters installed by a local Distributor Network Operator (DNO) in 76 homes during one month. The input dataset includes the active and reactive power recorded each hour. In order to map the real data to the proposed MG structure, the input data is randomly grouped into the 4 internal nodes of the AC $n G$ and the time domain signals are generated assuming a constant supply frequency of $50 \mathrm{~Hz}$. For the line model, each line is assumed to be $500 \mathrm{~m}$ long, with a line reactance to resistance ratio, $X / R=0.32$, and neglecting the cross-coupling between phases. The results for the real-time PF compared to the actual measured data is shown in Table III. As it can be seen, both the real-time signal processing $\left(V_{x}\right)$ and the PF output $\left(\widehat{V}_{x}\right)$ converge to remarkable close values. The proposed system is being used as the management software for our microgrid lab. Fig. 13 shows the front-end power converter used as the interface to the main AC grid, the 3P-SST and the energy storage system installed at the lab. The developed application runs under Windows 8 on a server acting as the central controller. The host server is a virtualized environment under VMware ESXi 5.5. 


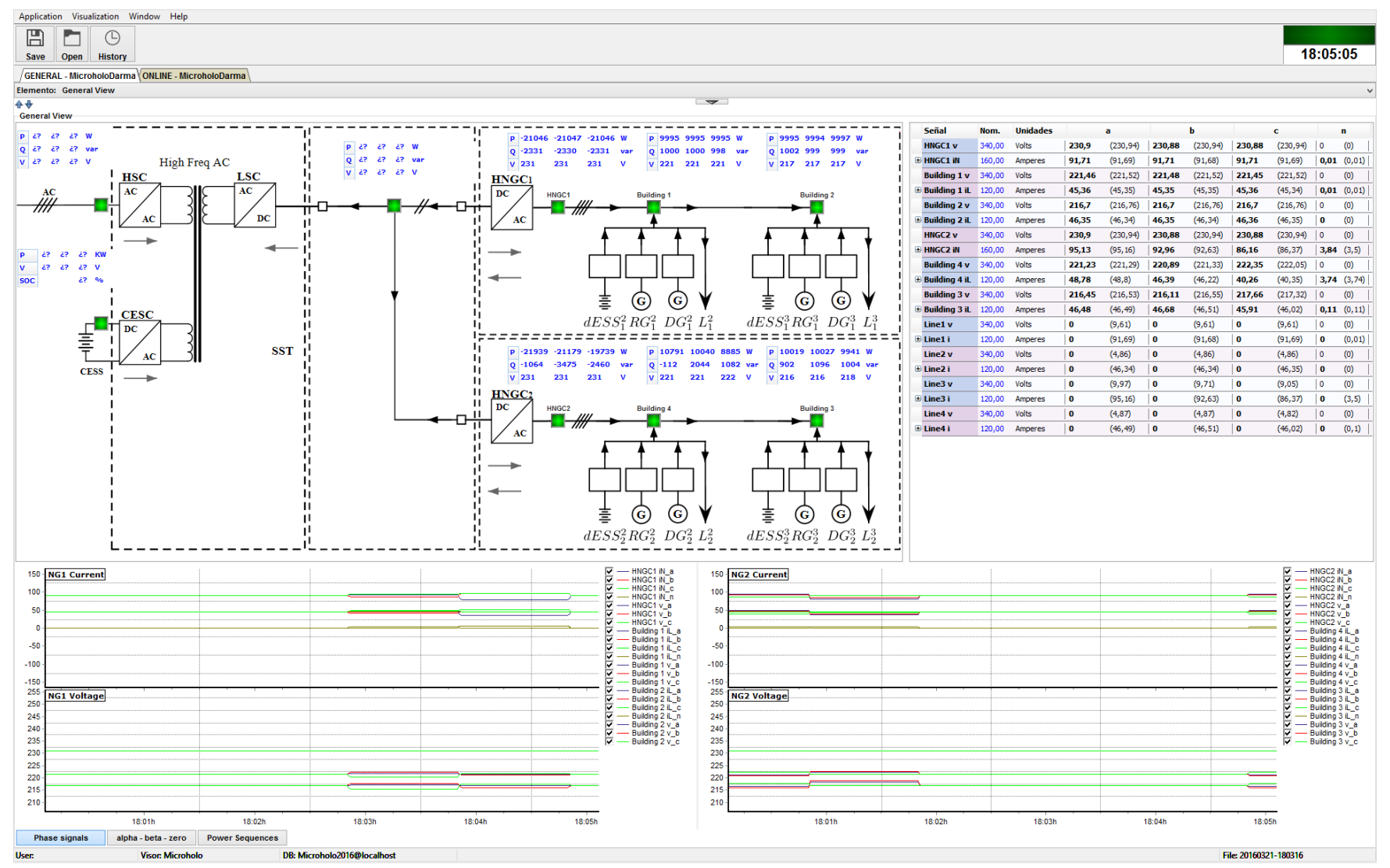

Fig. 12. Developed application running with the DNO emulated test data.

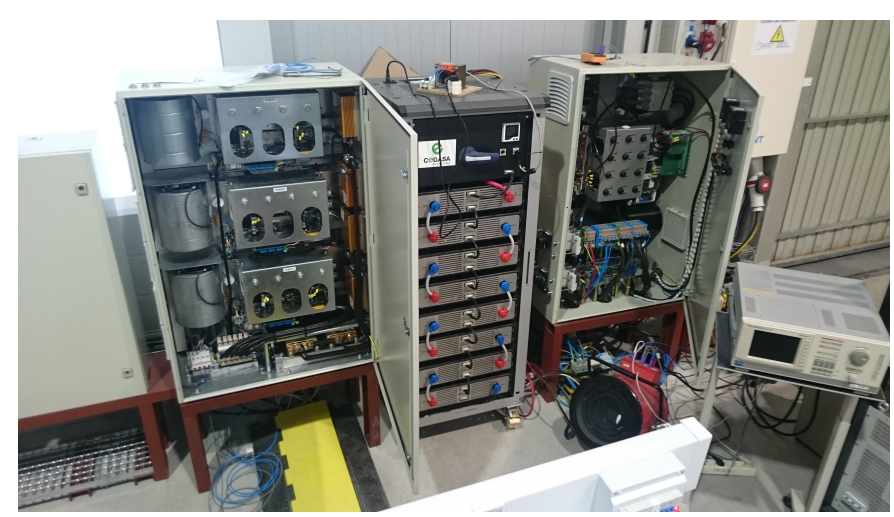

Fig. 13. Main power converters of the microgrid lab used for the system testing.

\section{CONCLUSION}

This paper has presented a monitoring and control system for an alternative distribution grid based on the use of a 3PSST with ESS. The work includes the explanation for the control of the 3P-SST and the AC $n G$. The developed system includes the realization of a distributed signal processing framework which allows to calculate in real time, with a time-frame of $200 \mathrm{~ms}$, the PF on the grid. The proposed digital processing chain relies on the Goertzel algorithm in order to obtain the grid voltages/currents $p n z$ sequences. The communication system allows sending sampled-values data between the control units and the local processing. The system has a relative low burden for the communications network and can be scaled to a higher number of nodes. An application running on a lab environment has been built for demonstration purposes.

\section{REFERENCES}

[1] S. Pourmousavi and M. Nehrir, "Real-time central demand response for primary frequency regulation in microgrids," Smart Grid, IEEE Transactions on, vol. 3, no. 4, pp. 1988-1996, Dec 2012.

[2] N. Tummuru, M. Mishra, and S. Srinivas, "Dynamic energy management of renewable grid integrated hybrid energy storage system," Industrial Electronics, IEEE Transactions on, vol. 62, no. 12, pp. 7728-7737, Dec 2015.

[3] S. Adhikari and F. Li, "Coordinated V-f and P-Q control of solar photovoltaic generators with MPPT and battery storage in microgrids," Smart Grid, IEEE Transactions on, vol. 5, no. 3, pp. 1270-1281, May 2014.

[4] J. Guerrero, M. Chandorkar, T. Lee, and P. Loh, "Advanced Control Architectures for Intelligent Microgrids-Part I: Decentralized and Hierarchical Control," IEEE Transactions on Industrial Electronics, vol. 60, no. 4, pp. 1254-1262, apr 2013.

[5] J. M. Guerrero and P. C. Loh, "Advanced Control Architectures for Intelligent Microgrids-Part II: Power Quality, Energy Storage, and AC/DC Microgrids," Industrial Electronics, IEEE Transactions on, vol. 60, no. 4, pp. 1263-1270, 2013.

[6] O. Palizban and K. Kauhaniemi, "Microgrids in active network management-Part I: Hierarchical control, energy storage, virtual power plants, and market participation," Renewable and Sustainable Energy Reviews, vol. Volume 36, no. August 2014, pp. 428-439, 2014.

[7] V. C. Gungor, D. Sahin, T. Kocak, S. Ergut, C. Buccella, C. Cecati, and G. P. Hancke, "Smart Grid Technologies: Communication Technologies and Standards," IEEE Transactions on Industrial Informatics, vol. 7, no. 4, pp. 529-539, nov 2011. 
[8] F. M. Cleveland, "IEC 61850-7-420 communications standard for distributed energy resources (DER)," in Power and Energy Society General Meeting-Conversion and Delivery of Electrical Energy in the 21st Century, 2008 IEEE. IEEE, 2008, pp. 1-4.

[9] A. Vaccaro, M. Popov, D. Villacci, and V. Terzija, "An Integrated Framework for Smart Microgrids Modeling, Monitoring, Control, Communication, and Verification," Proceedings of the IEEE, vol. 99, no. 1, pp. 119-132, jan 2011.

[10] V. C. Gungor, D. Sahin, T. Kocak, S. Ergut, C. Buccella, C. Cecati, and G. P. Hancke, "A Survey on Smart Grid Potential Applications and Communication Requirements," IEEE Transactions on Industrial Informatics, vol. 9, no. 1, pp. 28-42, feb 2013.

[11] N. L. Diaz and T. Dragicevic, "Intelligent Distributed Generation and Storage Units for DC Microgrids-A New Concept on Cooperative Control Without Communications Beyond Droop Control," Smart Grid, IEEE Transactions on, vol. 5, no. 5, pp. 2476-2485, 2014.

[12] Q. Fu, L. F. Montoya, A. Solanki, A. Nasiri, V. Bhavaraju, T. Abdallah, and D. C. Yu, "Microgrid Generation Capacity Design With Renewables and Energy Storage Addressing Power Quality and Surety," IEEE Transactions on Smart Grid, vol. 3, no. 4, pp. 2019-2027, dec 2012.

[13] C. Li, T. Dragicevic, M. Garcia Plaza, F. Andrade, J. Vasquez, and J. Guerrero, "Multiagent based distributed control for state-of-charge balance of distributed energy storage in DC microgrids," in Industrial Electronics Society, IECON 2014 - 40th Annual Conference of the IEEE, Oct 2014, pp. 2180-2184.

[14] D. Wu and F. Tang, "A Control Architecture to Coordinate Renewable Energy Sources and Energy Storage Systems in Islanded Microgrids," Smart Grid, IEEE Transactions on, vol. 6, no. 3, pp. 1156-1166, 2015.

[15] D. Laverty, D. Morrow, R. Best, and P. Crossley, "Telecommunications for Smart Grid: Backhaul solutions for the distribution network," in 2010 IEEE Power and Energy Society General Meeting, jul 2010, pp. 1-6.

[16] S. Mohagheghi, J. Stoupis, and Z. Wang, "Communication protocols and networks for power systems-current status and future trends," in Power Systems Conference and Exposition, 2009. PSCE'09. IEEE/PES. IEEE, 2009, pp. 1-9.

[17] C. Ozansoy, A. Zayegh, and A. Kalam, "The Application-View Model of the International Standard IEC 61850," IEEE Transactions on Power Delivery, vol. 24, no. 3, pp. 1132-1139, jul 2009.

[18] T. S. Ustun, C. Ozansoy, and A. Zayegh, "Modeling of a Centralized Microgrid Protection System and Distributed Energy Resources According to IEC 61850-7-420," IEEE Transactions on Power Systems, vol. 27, no. 3, pp. $1560-1567$, aug 2012.

[19] D. M. E. Ingram, P. Schaub, R. R. Taylor, and D. A. Campbell, "Performance Analysis of IEC 61850 Sampled Value Process Bus Networks," IEEE Transactions on Industrial Informatics, vol. 9, no. 3, pp. 1445-1454, aug 2013.

[20] T. Morstyn, B. Hredzak, and V. Agelidis, "Distributed Cooperative Control of Microgrid Storage," IEEE Transactions on Power Systems, vol. 30, no. 5, pp. 2780-2789, sep 2015.

[21] F. Nejabatkhah and Y. W. Li, "Overview of Power Management Strategies of Hybrid AC DC Microgrid," IEEE Transactions on Power Electronics, vol. 30, no. 12, pp. 7072-7089, dec 2015.

[22] X. Yu, X. She, X. Ni, and A. Huang, "System integration and hierarchical power management strategy for a solid-state transformer interfaced microgrid system," IEEE Transactions on Power Electronics, vol. 29, no. 8, pp. 4414-4425, Aug. 2014.

[23] Y. Ju, W. Wu, B. Zhang, and H. Sun, "An extension of FBS three-phase power flow for handling pv nodes in active distribution networks," Smart Grid, IEEE Transactions on, vol. 5, no. 4, pp. 1547-1555, July 2014.

[24] A. Dunkels, "uip (micro ip)." [Online]. Available: http://dunkels.com/adam/

[25] G. Goertzel, "An algorithm for the evaluation of finite trigonometric series," The American Mathematical Monthly, vol. 65, no. 1, pp. 34-35, 1958.

[26] W. Duesterhoeft, M. W. Schulz, and E. Clarke, "Determination of instantaneous currents and voltages by means of alpha, beta, and zero components," American Institute of Electrical Engineers, Transactions of the, vol. 70, no. 2, pp. 1248-1255, July 1951.

[27] G. C. Paap, "Symmetrical components in the time domain and their application to power network calculations," Power Systems, IEEE Transactions on, vol. 15 , no. 2, pp. 522-528, 2000.

[28] P. Arboleya, C. Gonzalez-Moran, and M. Coto, "Unbalanced power flow in distribution systems with embedded transformers using the complex theory in alpha beta 0 stationary reference frame," IEEE Transactions on Power Systems, vol. 29, no. 3, pp. 1012-1022, 2014.

[29] P. Arboleya, B. Mohamed, C. Gonzalez-Moran, and I. El-Sayed, "BFS algorithm for voltage-constrained meshed dc traction networks with nonsmooth voltage-dependent loads and generators," Power Systems, IEEE Transactions on, vol. PP, no. 99, pp. 1-11, 2015.

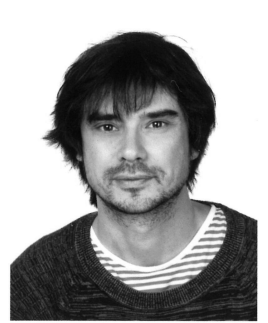

Pablo García (S'01-A'06-M'09) received the M.S. and $\mathrm{PhD}$ degrees in electrical engineering and control for the University of Oviedo, Gijón, Spain in 2001 and 2006 respectively. For the period of 2002-2006 he has been awarded a fellowship of the Personnel Research Training Program funded by the Spanish Ministry of Education. In 2004 he was a visitor Scholar at the University of MadisonWisconsin at the Wisconsin Electric Machines and Power Electronics Consortium (WEMPEC). He is the coauthor of more than 20 IEEE journals and 30 international conferences. He is currently an associate professor with the Department of Electrical, Computer and Systems Engineering, University of Oviedo. His research interest includes control of Grid-tied converters, solid-state transformers, energy conversion, AC drives, sensorless control, AC machines diagnostics, and signal processing.

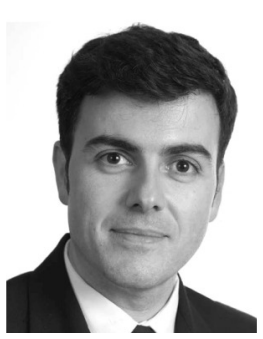

Pablo Arboleya (SM'13) received the M.Sc. and $\mathrm{Ph} . \mathrm{D}$. (with distinction) degrees from the University of Oviedo, Gijón, Spain, in 2001 and 2005, respectively, both in Electrical Engineering. Nowadays, he works as Associate Professor in the Department of Electrical Engineering at the University of Oviedo (with tenure since 2010). Presently his main research interests are focused in the micro-grid and smart-grid modelling and operation techniques, railway traction networks simulation and combined AC/DC power flow algorithms.

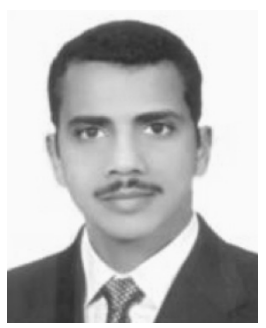

Bassam Mohamed received the M.Sc degree from the University of Oviedo, Gijón, Spain, in 2014. $\mathrm{He}$ is now pursuing his Ph.D studies in the Department of Electrical Engineering at the University of Oviedo. His master thesis was focused on implementing power flow and optimal power solver for transmission networks. Now, he is working on modelling and simulation of AC and DC micro-grid and railway traction networks.

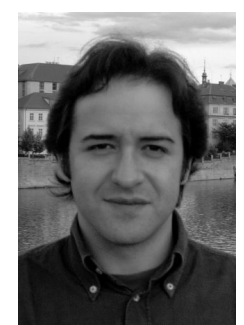

Abel A. Cuadrado Vega (S'02-A'04-M'05) received the M.S. and Ph.D. degrees in electronic and control engineering from the University of Oviedo, Gijón, Spain, in 1998 and 2003 respectively, being also a recipient of the University of Oviedo Outstanding $\mathrm{PhD}$ Thesis Award. He was a visiting researcher at the Betriebsforschungsinstitut (BFI) in Düsseldorf, Germany, in 2005. He is currently an Associate Professor with the Department of Electrical, Electronic, Computer and Systems Engineering of the University of Oviedo, teaching control theory and automation. His research interests are focused on supervision of complex industrial processes, specially those related to electric power generation and the steel industry.

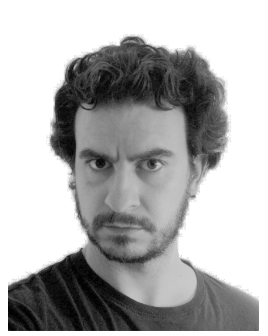

Marcos Cuadrado Vega received the B.S. degree in computer science from the University of Oviedo, Gijón, Spain, in 2002. He currently works as software developer and IT administrator in the Department of Electrical, Computer and Systems Engineering at the University of Oviedo. 